\title{
Characterization of a focal plane array (FPA) infrared camera.
}

\author{
by H. Pron, W. Menanteau, C. Bissieux, J.L. Beaudoin
}

Université de Reims, Unité de Thermique et Analyse Physique, Laboratoire d'Energétique et d'Optique, UFR Sciences,

Moulin de la Housse, BP 1039, 51687 Reims Cedex 2, France, e-mail: herve.pron@univ-reims.fr

\begin{abstract}
:
FPA infrared cameras are presently at a fast development stage, owing to the rapid increase of the computers performances but, up today, only a few works have dealt with the characterization of such equipments.

We present here the results of our first characterization tests, realised with a long wave FPA camera (CEDIP IRC 320-4 LW). Our study was led according to three directions : spatial resolution (in order to point out the correlation between neighbouring pixels), temporal resolution (as a function of the windowing of the matrix) and, finally, thermal resolution.
\end{abstract}

\section{Introduction}

The present study deals with the characterization of a CEDIP IRC 320-4 LW Focal Plane Array camera according to the three following points of view : spatial resolution, temporal resolution and thermal resolution.

First, a Slit Response Function (SRF) was realised with a standard lens, in order to determine the minimal size of an object allowing a good measurement of its luminance. Then, the results have been transposed in terms of projected image on the matrix of detectors, so that the spatial resolution can be expressed as a minimal number of pixels leading to a correct measurement.

In the second time, we studied the behaviour of the system from a temporal point of view, in particular the relation between the sub-windowing of the array and the frequency limit.

Last but not least, the intrinsic noise (expressed in digitised values) of the system has been estimated ; this noise, together with a thermal calibration, allowed the estimation of the Noise Equivalent Temperature Difference (NETD).

\section{Spatial characterization : Slit Response Function (SRF)}

The CEDIP "IRC 320-4 LW" camera is a long wave Focal Plane Array (FPA) camera built on a matrix of $320 \times 240$ detectors ; the size of each detector is $25 \mu \mathrm{m}$ and the pitch $30 \mu \mathrm{m}$.

Very well-defined images can be realised with this camera, more especially by the use of a "magnification $\times 1$ " lens. Figure 1 presents an example of an image realised with such a lens : the lines of a strain gauge, which are about $35 \mu \mathrm{m}$ large, can be clearly distinguished; but would the luminance measurement on so thin details be correct ?

In order to obtain an objective value for the spatial resolution, we carried out the following experiment : a thermal slit, the width of which can be controlled, was put in front of the FPA camera (equipped with a $50 \mathrm{~mm}, 20^{\circ}$ lens) at a distance of $660 \mathrm{~mm}$. A first image was taken with the slit wide open; then, the slit has been progressively closed and images were recorded for several different widths. The amplitude was then calculated for each width of the slit and normalised compared to the maximal value (obtained with the slit wide opened).

Figure 2 presents the results obtained for this Slit Response Function : a value of $95 \%$ for SRF is obtained for a slit of $1.5 \mathrm{~mm}$ width; according to the distance between the slit and the camera, this limit corresponds to $0.65 \%$ of the field of view, that is about two pixels in the plane of the FPA. 
As a conclusion, it is necessary to remember that, on the one hand, a high quality of the image is not necessarily synonymous with high accuracy of the measurement and, on the other hand, a good measurement can be realised if the dimensions of the object are about $1 \%$ of the field of view.

\section{Temporal aspects}

\subsection{Time resolution}

The CEDIP IRC320-4LW is a "snap shot" camera, that means that all detectors are exposed to the radiation of the observed infrared scene at the same time. This first duration is called "integration time" $t_{i}$ (adjustable from 10 $\mathrm{s}$ up to a few milliseconds). Then, the signal is read by a four-channel Data Acquisition Card, at a rate $f_{D A C}$ of $1,25 \mathrm{MHz}$ per channel; so that the "reading time" $t_{r}$ can be expressed as:

$$
t_{r}=\frac{n_{l} \times n_{c}}{4 \times f_{D A C}}
$$

where $n_{l}$ is the number of lines and $n_{c}$ the number of columns.

The last period, called "Settle Time" is dedicated to the re-initialisation of the system and needs at least $15 \mu \mathrm{s}$.

The frame period is thus:

$$
t_{i}+t_{r}+t_{s}=t_{i}+\frac{n_{l} \times n_{c}}{4 \times f_{D A C}}+t_{s}
$$

The minimal frame period is thus:

$$
\begin{aligned}
& t_{\text {min }}=10 \times 10^{-6}+\frac{320 \times 240}{4 \times 1.25 \times 10^{6}}+10 \times 10^{-6}=15.385 \times 10^{-3} \mathrm{~s} \\
& \Rightarrow f_{\max }=64.998 \mathrm{~Hz}
\end{aligned}
$$

Experimentally, we established that no synchronised image could be obtained at $65 \mathrm{~Hz}$; this result rather well agrees with the calculated value.

Figure 3 shows the relative values of these three terms for a few different frame frequencies. Obviously, the maximal frame frequency (64 Hz for a full window) can not be significantly increased by reducing the Integration Time or the Settle Time. Consequently, the only way to reach high frame frequencies consists in reducing the number of data to acquire, i.e. reducing the size of the array. Figure 4 shows the evolution of the frame frequency limit as a function of the array surface (in pixels). For example, a matrix of 50x50

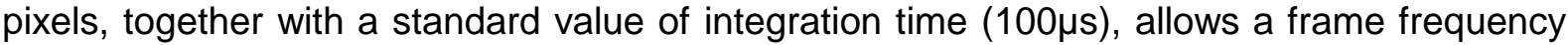
reaching $1626 \mathrm{~Hz}$; under the same conditions, a frame frequency of $3174 \mathrm{~Hz}$ can be obtained with an array limited to $200 \times 5$ pixels. Experimentally, we tested the system up to $3 \mathrm{kHz}$ with these dimensions of array, and no problem occurred.

\subsection{Influence of the integration time on the infrared signal}

The integration time is adjustable between $10 \mu$ s and some milliseconds; low-energy infrared scenes can be imaged by using long integration times, whereas very rapid phenomena would imply the use of a short integration time. However, the greatest care must be taken in each case.

On the one hand, short integration times lead to a low ratio measurement duration / frame period, which means that most of the time, the camera does not share at the scene : an integration time of $100 \mu \mathrm{s}$ corresponds to only $0.65 \%$ of the frame period at $65 \mathrm{~Hz}$ and $10 \%$ of the frame period at $1000 \mathrm{~Hz}$. Figure 5 presents the evolution of the ratio integration time I frame period as a function of the frame frequency, for a $100 \mu$ s integration time. For 
comparison, a scanning camera is staring at the scene (even if it is at different points) more than $50 \%$ of the frame period. This difference could be a disadvantage for FPA cameras.

On the other hand, long integration times can distort a non-stationary infrared signal. For example, figure 6 shows how a sinusoidal signal will be described by such a camera. The signal acquired between the instants $t$ and $t+t_{i}$ is assigned to the instant $t$ by the acquisition system and can be expressed by:

$$
\begin{aligned}
S \propto A \int_{t}^{t+t_{i}} \cos (\omega t+\phi) d t & =\frac{A}{\omega}[\sin (\omega t+\phi)]_{t}^{t+t_{i}}=\frac{A}{\omega}\left(\sin \left(\omega\left(t+t_{i}+\phi\right)-\sin (\omega t+\phi)\right)\right. \\
\Rightarrow \quad S & \propto \frac{2 A}{\omega} \sin \left(\frac{\omega t_{i}}{2}\right) \cos \left(\omega t+\frac{\omega t_{i}}{2}+\phi\right)
\end{aligned}
$$

If the value of $\frac{\omega t_{i}}{2}$ is small enough, then :

$$
S \propto t_{i} A \cos (\omega t+\phi)
$$

The relative error on the amplitude is :

$$
\left\|\frac{\omega t_{i}}{2 \sin \left(\omega t_{i} / 2\right)}-1\right\|
$$

The phase shift introduced is:

$$
\frac{\omega t_{i}}{2}=\pi f t_{i}
$$

For example, the relative error on the amplitude is less than $1 \%$ if the ratio integration time / period of the signal is less than $1 /(4 \pi)$; but, under these conditions, the phase error would be about 0.25 radians (more than $14^{\circ}$ ).

So, the integration time can introduce some systematic errors in the results. The greatest care must be taken in increasing the value of this parameter which determines, together with the Shannon criterion, the limit in frequency for lock-in thermography.

\section{Thermal calibration and thermal resolution}

\subsection{Thermal calibration}

Three parameters have to be taken into account : gain, offset and integration time. The Data Acquisition Card (DAC) gives a numerical value $V$ as a function of the input signal :

$$
V=\mathfrak{I}\left[10^{(G / 20)}\left(\frac{\text { Video }}{2}-\text { Offset }\right) \times 4096+8192\right]
$$

where the symbol $\mathfrak{I}$ represents the integer part of the value in the square brackets.

This expression can be easily inverted, in order to calculate the video signal issued from the detector as a function of the numerical value given by the system. As far as the results still depend on the integration time, we have tested it for different values of this parameter and for different temperatures. Figure 7 shows the dependence of the video signal versus integration time : the behaviour is linear until saturation (Figure 8).

$$
\text { Video }=L \times t_{i}+M \text {, }
$$

Besides, two new quantities can be introduced : the video signal per unit of integration time $L$, which corresponds to the slope of the curve, and the offset $M$ corresponding to the video value for an integration time equal to zero.

The values of the parameter $L$ corresponding to the curves of figure 8 are presented as functions of target temperature on figure 9; the points correspond to the experimental values and the continuous line has been obtained with the following model : 
with : $R=157.3 \quad B=900.9 \quad F=4.3$

$$
L=\frac{R}{e^{\frac{B}{T}}-F}
$$

These calibration constants $\mathrm{R}, \mathrm{B}, \mathrm{F}$ have been obtained by repeating the same procedure (calculation of the video signal, then of the ratio video signal / integration time) for several configurations of parameters (gain, offset and integration time) and for different temperatures. Then, a non-linear Gauss identification procedure, based on comparison between experimental and calculated values of $L$, taking into account the sensitivity of the model to each parameter, has been applied.

However, this thermal calibration needs to be limited to the linear area of the curves "video signal as a function of integration time and temperature" (figure 8); so, a validity domain has been defined (figure 10): integration times shorter than $20 \mu \mathrm{s}$ must be avoided and high temperatures can not be measured with long integration times (saturation of the detectors or of the DAC).

\subsection{Estimation of the N.E.T.D.}

The last important information concerning an infrared camera is its thermal resolution. In order to estimate this characteristic of the system, we realised a study in two steps : first, the intrinsic noise of the whole system (the values given by the manufacturer only concerns the detectors) was estimated; then a thermal calibration was realised, and the thermal resolution calculated.

A quite simple experiment leads to a good estimation of the noise : an image averaged on $N$ frames is realised and subtracted to a single image. The value $V_{i}$ of each pixel $i$ of an image is the sum of the signal $S_{i}$ and a noise $B_{i}$ :

$$
V_{i}^{1}=S_{i}+B_{i}
$$

The calculated average image obtained from series of $N$ images is less noisy :

$$
V_{i}^{N}=S_{i}+\frac{B_{i}}{\sqrt{N}}
$$

so that the value of each pixel of the "noise image" can be estimated by :

$$
B_{i}=\frac{\sqrt{N}}{\sqrt{N}-1}\left(V_{i}^{1}-V_{i}^{N}\right)
$$

An experimental estimation of the noise has been carried out, and the results converted in terms of Noise Equivalent Temperature Difference (NETD) owing to the thermal calibration above mentioned. Then, the average value has been calculated on the whole noise image.

The experimental average value for N.E.T.D. obtained for a "standard" configuration (gain: $12 \mathrm{~dB}$, offset: $1350 \mathrm{mV}$, integration time: $100 \mu \mathrm{s}$ ) is $21 \mathrm{mK}$; this result is to be compared to the value given by the constructor which is $18 \mathrm{mK}$ for a detector alone. The difference can be imputed to the amplification device and the acquisition system (electronic noise but also quantification noise).

Finally, it is important to note that the NETD is not a constant value.

On the one hand, we present here an average value, but each pixel has its own NETD. Moreover, the non-uniformity corrections have a great influence on the NETD, depending on the level of the observed scene compared to the levels of the scenes which have been used to realise the corrections (see figure 11); for that reason spatial NETD is not relevant.

On the other hand, the noise of the system will decrease as the integration time increases, as shown on figure 12. 


\section{Conclusions}

As a conclusion, it can be stated that Focal Plane Array cameras can be used as measurement devices provided that some conditions are carefully checked.

First, these cameras present a very good spatial resolution and the cross-talk between neighbours seems to be limited. On the temporal point of view, frame frequencies higher than one kilohertz can be obtained by reducing the size of the array; nevertheless, the user must be careful about the influence of the integration time: too short, it could prevent from catching a brief phenomenon, too long, it could deform a non-stationary signal. Finally, the thermal resolution of such cameras is very high, but depend on the quality of the matrix nonuniformity corrections.
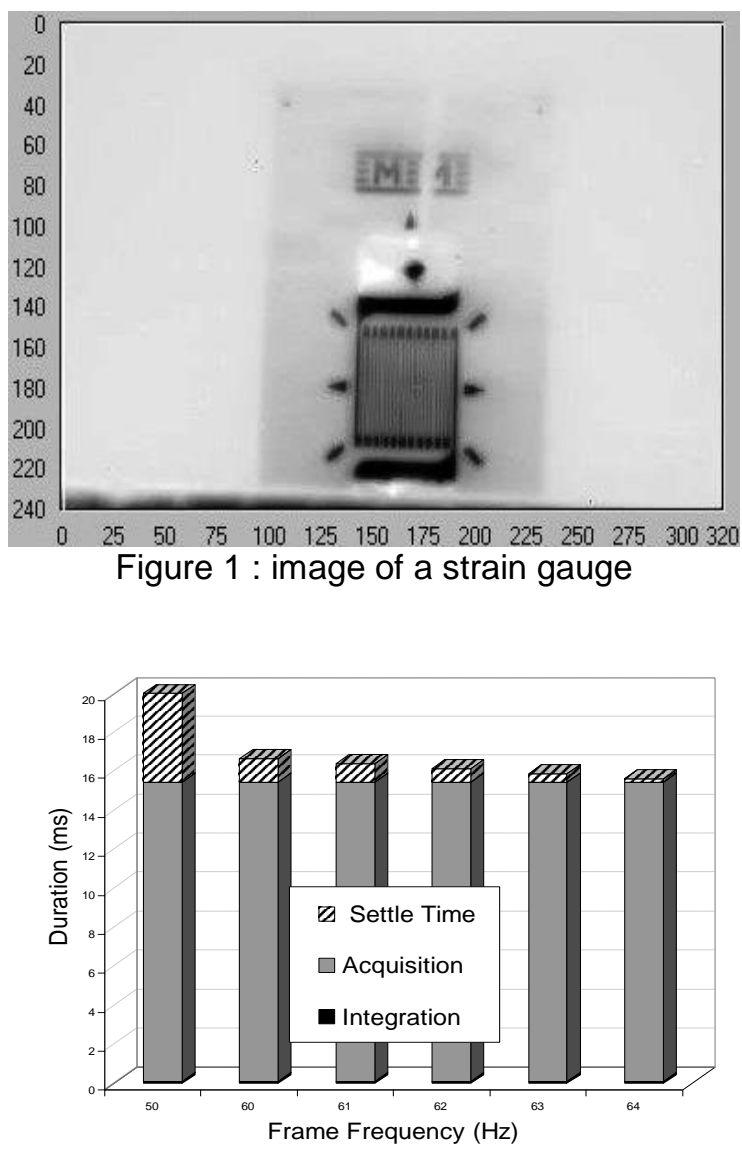

Figure 3 : Evolution of integration time, acquisition duration and settle time vs. frequency

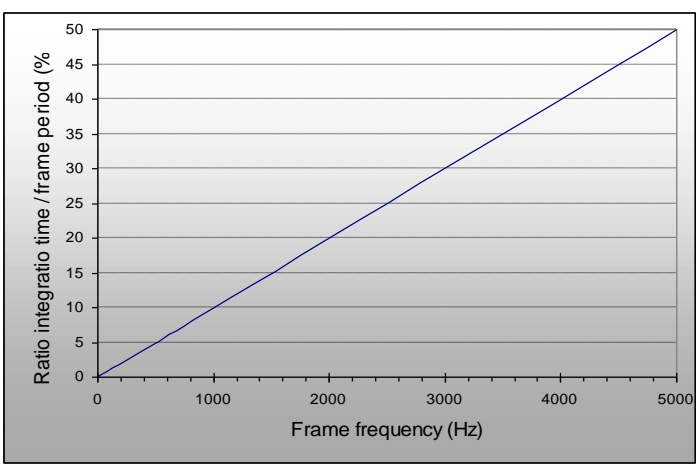

Figure 5 : Evolution of the ratio integration time / frame period as a function of the frame frequency

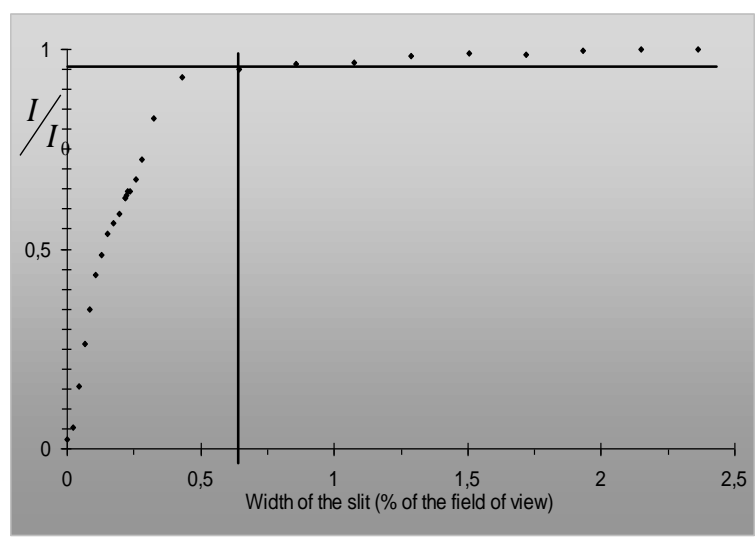

Figure 2 : SRF of the CEDIP IRC 320-4 LW camera, for a slit at $660 \mathrm{~mm}, 20^{\circ}$ lens

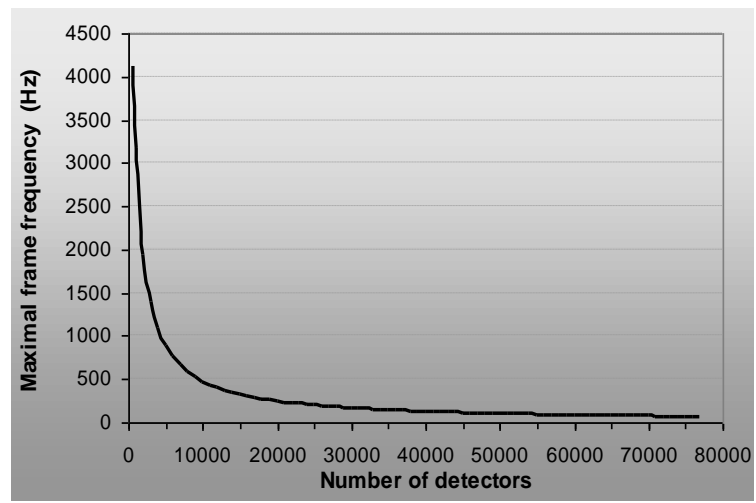

Figure 4 : Evolution of the maximal frame frequency as a function of the windowed array

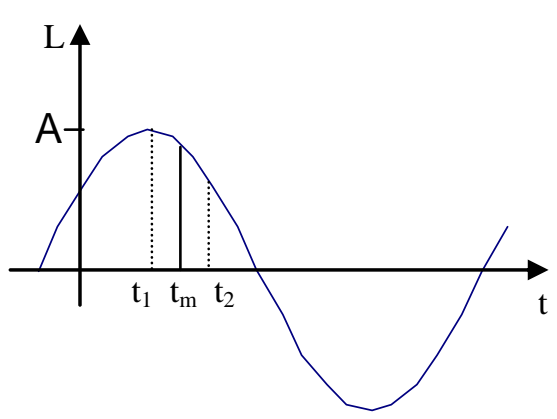

Figure 6 : Influence of the integration time on a sinusoidal signal 


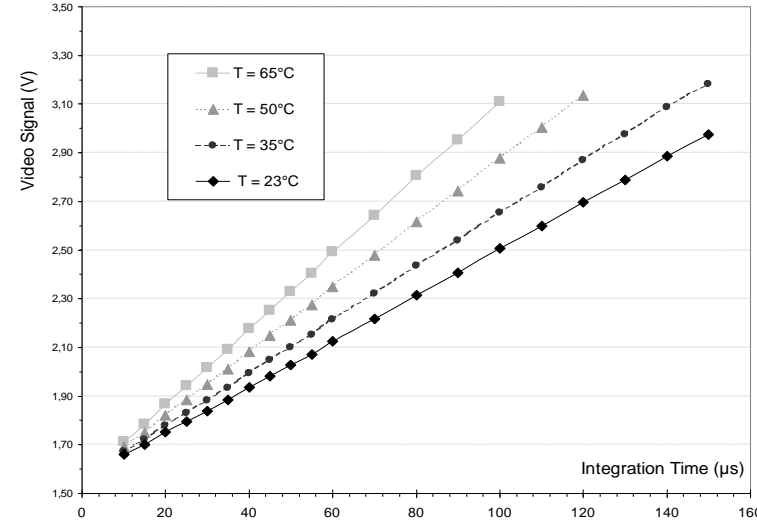

Figure 7 : Evolution of the video signal with the integration time, for different target temperatures

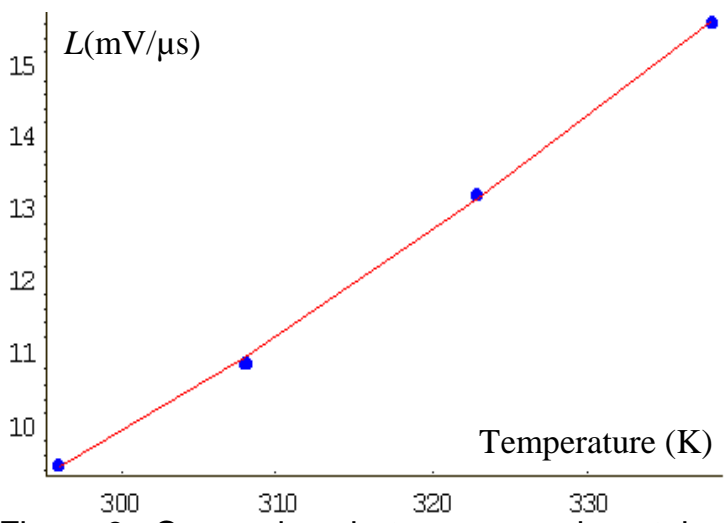

Figure 9 : Comparison between experimental and identified values of $L$

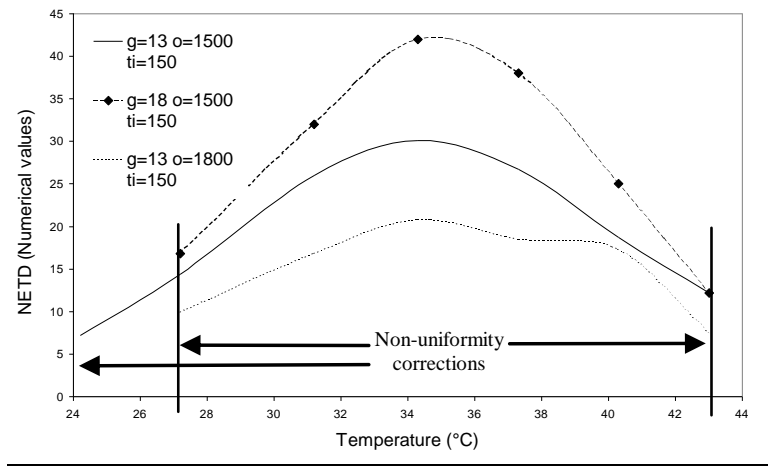

Figure 11 : Non-Uniformity Corrections and NETD

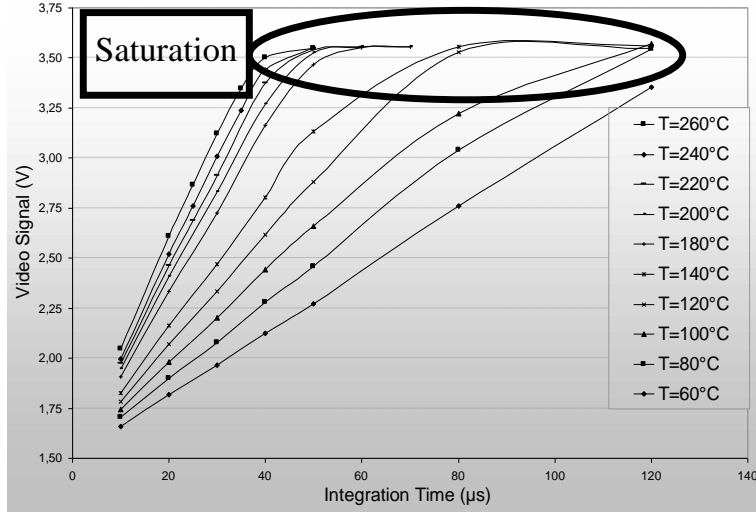

Figure 8 : Saturation of the video signal for high temperatures and/or long integration times

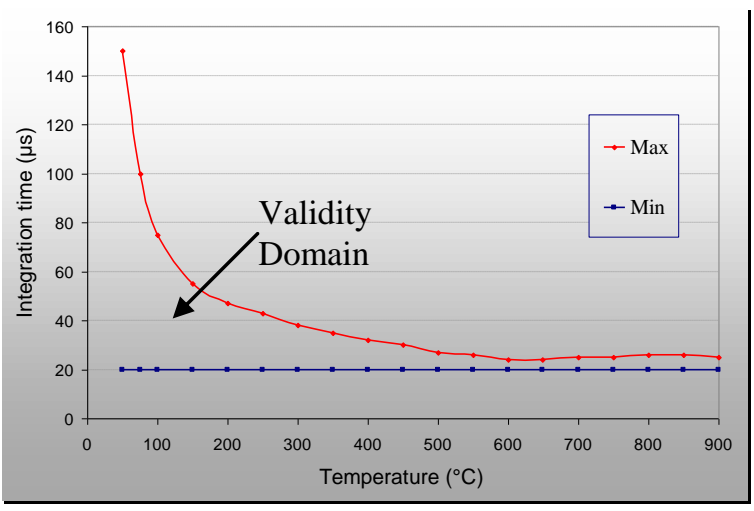

Figure 10 : validity domain of the thermal calibration

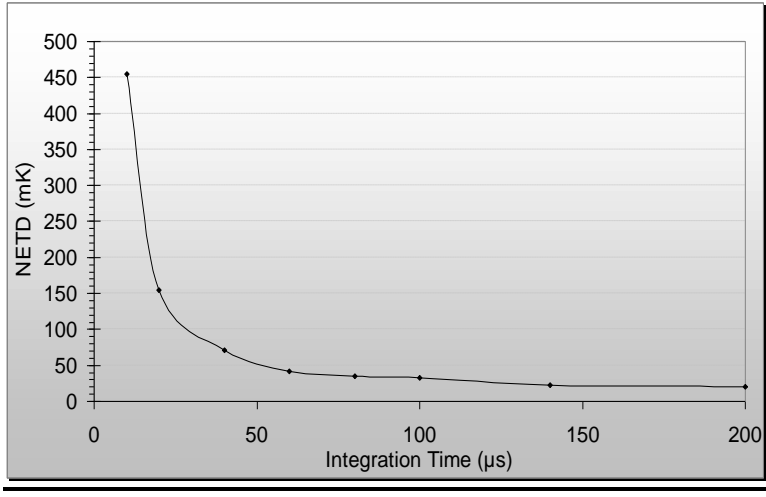

Figure 12 : influence of integration time on NETD 